\title{
Characterization of a High Resolution Acquisition System for Marine Geophysical Applications
}

\author{
S. Shariat Panahi (1), F. Alegria (2), A. Manuel (1)
}

(1) Vilanova i la Geltrú Technological Center - Technical University of Catalonia (UPC)

Rambla Exposicio s/n, 08800, Vilanova I la Geltru, Spain

(2) Technical University of Lisbon (IST), Av. Rovisco Pais, 1049-001, Lisbon

\section{Introducción}

The progress of the marine instrumentation in the last decade is continuously bringing up new goals as higher autonomy, lower power consumption and better signal quality, to be achieved by the engineers.

The quality of the signal is one the main issues related to acquisition systems built for marine geophysical applications where the signal level can be very low depending on the depth of instrument operation. In this field, Ocean Bottom Seismometers (OBS) are widely used for the study of the seabed sub-layers of a certain area when instruments are deployed on the sea bottom, at the depth of up to $6000 \mathrm{~m}$, where they collect data for about 3 months. They are more used in active seismicity where the artificial source is a compressed air-gun dragged by the oceanographic vessel at low depth, generating acoustic wave fronts every certain time, that travel to the bottom of the ocean and reflect and refract by the different sub-layers before being acquired by the OBS. The equipment uses three sensors: a tri-axial geophone that collects seabed vibrations, a hydrophone that collects water pressure data and an acoustic transducer for the recovery process that starts by reception of a signal generated by the deck unit on the ship, releasing the attached weight and allowing the OBS to rise up to the surface due to its structural floatability.

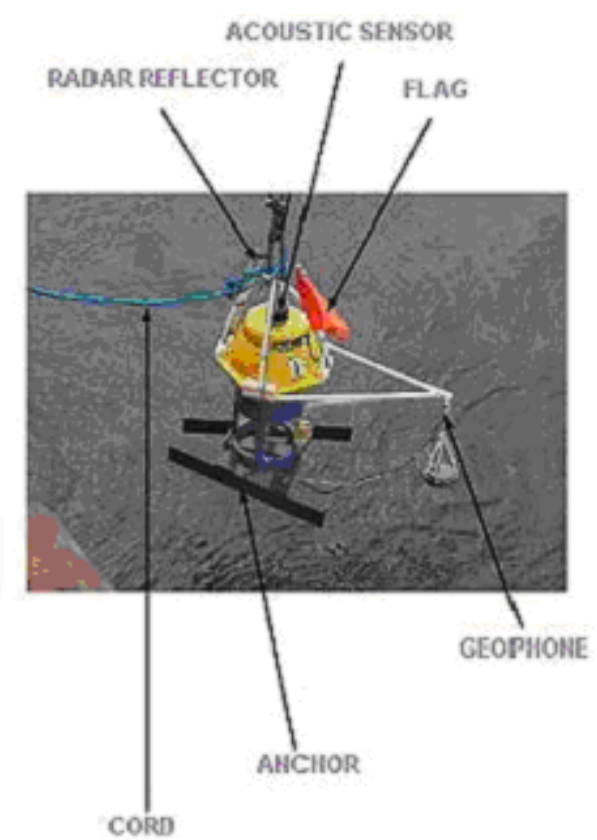

Figure 1: Ocean Bottom Seismometer (OBS)
In active seismicity, the maximum operation depth of the OBS places the equipment more than $6000 \mathrm{~m}$ away from the acoustic source, reducing the signal level received by the instrument and increasing the need of a high Signal to Noise Ratio(SNR), high resolution and high Dynamic Range(DR) acquisition system [6]. This paper presents the characterization of a four channel, 24 bits acquisition system, designed and built for marine geophysical applications, where different tests based on "IEEE-STD-1057 Standard for Digitizing Waveform Recorders" and "IEEE-STD-1241 Standard for Terminology and Test Methods" have been carried out to calculate the main parameters of the system taking into account its final application.

\section{The Acquisition system}

The acquisition system is based on a 4 channel 24 bits CS5372/76 analog to digital conversion (ADC) together with input signal amplification. The CS5372 are two channel high dynamic range, fourth order $\bullet-\bullet$ modulators designed for geophysical and sonar applications. Used in combination with CS5376 digital filter, a unique high resolution $A / D$ measurement system results which all together provide higher dynamic range of $124 \mathrm{~dB} @ 411 \mathrm{~Hz}$ bandwidth and a Total Harmonic Distortion(THD) of $-118 \mathrm{~dB}$ [3], while consuming low power per channel. The modulators generate an oversampled serial bit stream at 512kbits per second when operated from clock frequency of $2.048 \mathrm{MHz}$.

The input stage of the acquisition system is based on OP97 and OP297 low noise amplifiers in a single ended to differential signal converter configuration, due to the fact that the signal coming from the geophone is single ended and the CS5372 ADC is differential. A MC68332 microcontroller is in charge of the configuration of the CS5376 digital filter selecting sampling rate, filter output and low power operations, through a QSPI (Queued Serial port interface) bus. All the necessary signals for the datalogger to function correctly have to be generated from a single crystal and being aware of the fact that in any marine seismic application, timing is of great importance, a Vectron OC260 $32.768 \mathrm{MHz}$ crystal is used offering a stability of $40 \mathrm{ppb}$ and an electronic module is designed and built integrating a ICS52701 phased locked loop generating different signal frequencies for the system. 


\section{Characterization tests}

The designed test programming is based on the graphical language LabVIEW 7.1, where a frequency analysis of the acquired data is performed. In order to characterize the acquisition system built, the following tests based on the IEEE-1057 and IEEE-1241 standards have been carried out:

Discrete Fourier Transform (DFT) test

Random noise test

Crosstalk test

Taking into account that the noise analysis is of great importance in any acquisition system aimed for geophysical applications and 24 bits resolution of the system under test, an ultralow noise and distortion Stanford Research System (SRS) DS630 function generator is used to generate the input signals and AA type batteries are used to supply the acquisition system. The DS630 function generator presents a $-110 \mathrm{~dB}$ Total Harmonic Distortion (THD) in $5 \mathrm{kHz}$ bandwidth and minimum $10 \mathrm{uVpp}$ amplitude allowing to characterize the system using low input amplitudes as in real conditions.

- DFT test [1][2]: A sine wave signal with known amplitude and frequency is applied at the input of the system and 4096 samples/channel with a sampling frequency of 250 sps are acquired and transferred to a PC, where the test program in LabVIEW stores the data in a data file. The DFT Analysis option of the test program reads the data file stored previously, calculating the DFT of the acquired data and the desired parameters from the DFT data for every channel separately. The Save Panel Image option allows the user to store the front panel data into a file for post-processing. As signal level received by the geophone can be very small due to the fact that the instrument can be deployed at more than $6000 m$ away from the artificial source, the total noise performance of the system results in a key feature of the overall equipment, therefore parameters as Signal-to-Noise Ratio (SNR), Dynamic Range (DR) and Effective Number of Bits (ENOB) have been calculated. In addition, to characterize the system nonlinearities, the calculation of parameters based on harmonic distortion has been included.

- Random Noise test [1][2]: In order to calculate the noise generated by the acquisition system circuitry, in this case by the input amplifier stage of the module, a random noise test has been implemented. All the channels under test are grounded and two separate acquisitions of 4096 samples/channel at 250 sps sampling frequency have been carried out and stored in two data files. The Random Noise Analysis option of the designed software reads the data files previously stored and by subtracting the data samples, eliminates the ADC fixed-pattern errors [4] that occur in the same location in successive records and calculates the noise variance.

$$
\sigma^{2}=\frac{1}{2 M} \sum_{n=1}^{M}\left(y_{a n}-y_{b n}\right)^{2}
$$

where 2 is the noise variance, yan and ybn are the data samples of successive acquisitions and $M$ is the number of samples/channel. The Save Panel Image allows to store the final results for post-processing.

- Crosstalk test [1][2]: The calculation of crosstalk level of a multi-channel acquisition system is a good evaluation of the PCB design of the module, as this parameter is result of Electromagnetic Interference (EMI) between different parts of the board as well as the ground and power supply plane problems. A full-scale signal is applied to the channel under test while all the other channels are shielded and a 4096 samples/channel data acquisition is carried out and stored in a data file. The Crosstalk Analysis option of the designed software reads the data file stored previously and calculates the crosstalk level related to every channel using the following expression:

$$
\text { Crosstalk }(d B)=20 \log \left(\frac{R M S(\text { spunious })}{\text { RMS(signal) }}\right)
$$

\section{Results}

The need of a high-resolution system with a high Signal-to-Noise Ratio (SNR) to collect low level signals has taken us to use low level amplitude signals using a DS630 ultra-low function generator together with batteries for power supply of this module to reduce noise from external elements. The bandwidth of the system is defined by the selected sampling frequency as in CS5372/76, this is given by $40 \%$ of the sampling frequency, in this case $100 \mathrm{~Hz}$.

The $50 \mathrm{~Hz}$ line coupling through the input signal is corrected by eliminating the samples corresponding to this frequency and its successive harmonics, and replacing the eliminated samples by the last value before the $50 \mathrm{~Hz}$ interference peak set of DFT points. The number of DFT points to be eliminated at $50 \mathrm{~Hz}$ has to be adjusted by the user before obtaining the final result of the test. For low amplitude signals, the frequency of the input signal has to be introduced by the user as LabVIEW fundamental frequency detection functions do not operate below a certain signal amplitude level.

The characterization tests have been carried out over the system bandwidth $(100 \mathrm{~Hz})$ and the results of different parameters have been gathered in graphs. Figures 2 and 3 show the Dynamic Range (DR) and crosstalk results obtained during the implemented tests:

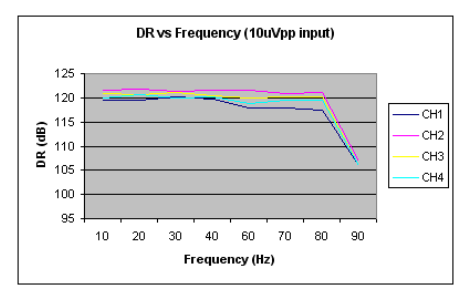

Figure 2: Dynamic Range (DR) results, sampling frequency: 250sps, number of samples/channel: 4096 


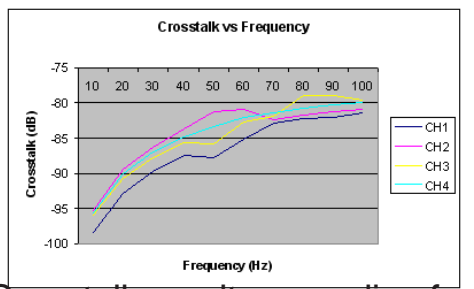

Figure 3: Crosstalk results, sampling frequency: 250sps, number of samples: 4096

These results show a $120 \mathrm{~dB}$ dynamic range over the system bandwidth, which means a reduction of $10 \mathrm{~dB}$ of the CS5372/76 dynamic range, while the crosstalk figures obtained in the test are $25 \mathrm{~dB}$ above the modulator specification value for below $10 \mathrm{~Hz}$ input signal frequency and about $40 \mathrm{~dB}$ above the same value for over $90 \mathrm{~Hz}$ input signal frequency. The noise analysis of the acquisition system has shown an Effective Number of Bits (ENOB) of about 18 bits that can be further optimised by using fully differential amplifiers as CS3301-2 with a noise figure of only $8.5 \mathrm{nV} / \mathrm{Hz}$. The use of fully differential amplifiers together with improvement of ground and power supply planes will improve the crosstalk results considerably [5].

\section{Conclusions}

The need of a high resolution and high signalto-noise ratio acquisition system in order to be used in deep ocean seismometers has taken us to design and implement a datalogger whose characterization tests have been explained in this paper. The implemented test results have shown a dynamic range of about $120 \mathrm{~dB}$ for below $80 \mathrm{~Hz}$ input signal frequency at $250 \mathrm{~Hz}$ sampling rate, leading to a 18bits of ENOB, while similar equipment available in the market offer $78 \mathrm{~dB}$ of dynamic range and 14 bits of ENOB at $200 \mathrm{~Hz}$ sampling rate.

However, noise features of the system can be further optimized by using CS3301-2 low noise fully differential amplifiers, that also improve the channel crosstalk levels detected during the tests.

\section{References}

[1] IEEE-STD-1057 Standard for Digitizing Waveform Recorders.

[2] IEEE-STD-1241 Standard for Terminology and Test Methods for Analog- to-Digital Converters.

[3] Cirrus Logic, "CS5371/72 low-Power high performance --・ modulators, 2001.

[4] Kent, H Lundberg. "Analog to Digital converter testing", 2002.

[5] Dallas-Maxim, "Effects of digital crosstalk in data converters", 2002.

[6] J. Basilio Simoes, Custodio Loureiro, Jorge Landeck, Carlos Correia, "Testing high resolution digitizers using conventional signal sources", Instrumentation Centre of the Physics Department, University of Coimbra, 1997.

\title{
Determination of the Confidence Interval for the ENOB of and ADC tested with the IEEE 1057 Random Noise Test
}

\author{
F. Corrêa Alegria( ${ }^{(1)}$, S. Shariat Panahi ${ }^{(2)}$, A. Manuel ${ }^{(2)}$, A. Cruz Serra ${ }^{(1)}$
}

(1) Instituto de Telecomunicações, Av. Rovisco Pais 1, 1049-001 Lisboa, Portugal Telefone: +351-218418485, Fax: +351-218417672, e-mail: falegria@lx.it.pt

(2) Vilanova i la Geltrú Technological Center - Technical University of Catalonia (UPC) Rambla Exposicio s/n, 08800, Vilanova I la Geltru, Spain.

\section{Introduction}

Analog to Digital (ADC) converters are nowadays extensively used in marine instrumentation. It brings several benefits to a data acquisition system like an efficient storage and processing of the gathered information. In certain applications, like the Ocean Bottom Seismometer (OBS) developed in Vilanova i la Geltrú Technological Center where the signals to be measured are very small, the importance in reducing noise in the acquisition system is paramount. One of the components that introduce noise is precisely the ADC.

Several tests exist to determine the noise produce by an ADC. One of them, described in the 1057 IEEE Waveform Digitizers Standard [1], is used to measure random noise and consists in shorting the ADC input and acquiring two sets of $M$ samples each ( $y a$ and $y b$ ) and subtracting, point by point the values of the samples in each record. The noise standard deviation, expressed in least significant bit units (LSB), is then calculated from the mean square values:

$$
\widehat{\sigma_{f}}=\sqrt{\frac{1}{2 M} \sum_{i=1}^{M}\left(y_{a w}-y_{b w}\right)^{2}}
$$

If the amount of random noise present is small compared with the quantization step, this method can not be used because the sample values will always be the same and the result will be 0 . To circumvent this problem, a small triangular stimulus signal should be applied to the input [1]. The start of the acquisition of the two sets should be triggered on the same level.

The amount of random noise may also be expressed in a parameter called Effective Number of Bits (ENOB). It states the number of bits of an ideal ADC with a quantization noise 